\title{
The effect of polarization charges on energy of univalent and bivalent donors in a spherical quantum dot
}

\author{
V.I.Boichuk ${ }^{1}$, I.V.Bilynskyi ${ }^{1}$, R.Ya.Leshko ${ }^{1}$ \\ 1 Department of Theoretical Physics, Ivan Franko Drohobych State Pedagogical University, \\ 24 Ivan Franko Str., 82100 Drohobych, Ukraine
}

Received March 4, 2008, in final form July 17, 2008

\begin{abstract}
The energy of electrons of univalent and bivalent impurity of a spherical $\beta$ - $\mathrm{HgS} / \mathrm{CdS}$ nanoheterostructure is calculated as a function of quantum dot radius by the variation technique in the case of finite and infinite wells in the effective mass approximation. The effect of polarization charges which arise at the separation boundary of the media is studied taking into account both the existence and the absence of an intermediate layer, where dielectric permittivity depends on the coordinate.
\end{abstract}

Key words: energy, impurity, polarization charges, potential well

PACS: $71.55 .-i, 73.21 . L a, 79.60 . J V$

\section{Introduction}

Intensive investigations of impurity states in different nanoscale systems have been reported in fundamental and applied publications for the recent 20 years. The use of effective mass approximation and of the dielectric continuum model yields good theoretical results that conform to experimental data.

The properties of impurities in quantum dots (QDs) are discussed [1-11]. For a univalent impurity in a spherical QD, exact solutions of the Schrödinger equation are obtained with extension over the Coulomb potential interaction of particles. The potential was taken as a rectangular spherical potential well due to a band mismatch at the separation boundary of the heterosystem. The Calculated results reveal that the values of quantum levels of a confined electron in a spherical QD can be quite different for the cases with finite and infinite well heights. The difference enhances if a QD size decreases. The structure of donor quantum levels is similar to the confined electron levels in a spherical QD if the well potential is stronger than that of a donor.

Based on exact solutions, quantum levels and energies of a donor located outside the center of a spherical QD are obtained using a variation technique [5]. Thus, the results are obtained for the ground and excited states of a donor in a spherical QD that is located inside or outside the QD. The results show that the splitting and the change of quantum level order are dependent on QD radius, impurity location, and well height. The splitting and the change of quantum level order are caused by the violation of the system symmetry and by the competition of Coulomb interaction and spatial confinement.

The dependence of energy of the system on the location of impurity was also studied [8]. It is shown that energies of optical transitions depend on QD sizes and location of an admixture center. By tuning QD sizes and the location of an admixture, it is possible for a heterosystem to emit light of a certain wavelength.

The fine structure of energy levels of a hydrogenic impurity located in the center of a spherical QD is calculated using a simple exact solution for a donor in a spherical QD [9]. It is mathematically shown that there is no QD radius at which it would be impossible to find the energy of the bound state of an impurity. The fine structure of an admixture is defined by perturbation theory. 
The corrections are calculated for the terms that describe spin-orbit coupling, correction for the relativistic kinetic energy and a Darwin term.

The effect of polarization charges of nanoheterostructure surface on the energy of a quasiparticle is investigated [12-14]. In these papers for heterosystems of different nature a presence of the bound surface charges at the separation boundary is taken into account with the help of potential of forces of electrostatic images. The conditions of the origin of the electron boundary states at the separation border are studied. The effect of the surface polarization charges of the heterostructure on the exciton energy and oscillator strength is defined.

Modern technology of composing semiconductor nanoheterostructures makes it possible to get nanosystems of sufficiently high quality. However, in the real conditions it is difficult to create a heterogeneous system with a stepwise change of all physical parameters at the separation boundary. Evidently, in the heterostructure there always exists a transitional layer, in which some physical parameter changes from its value in one semiconductor (dielectric) to the corresponding value in the other crystal. The effect of the surface transitional layer in which physical parameters are the functions of coordinates on the quasi-particle energy is discussed [15-18].

In order to analyse the separately performed investigations regarding donors and polarization charges, it is important to theoretically consider the effect of polarization charges on the univalent donor energy in a spherical QD. In [1-11] the attention was focused on the research of a univalent impurity. However, frequently the impurities in a QD have a valency greater than unity. Therefore, a theoretical study of the effect of polarization charges arising at the interface of the media on energy levels of a bivalent donor, as the simplest among polyvalent ones, that is dependent on QD sizes will be important for further detailed research of donors.

The work is aimed at determining the ground donor state energy of a univalent and a bivalent impurities in a spherical quantum dot with regard to polarization charges arising at the interface, taking into account both the existence and the absence of a transitional layer. Specific calculations are performed for the $\beta$-HgS/CdS nanoheterostructure.

\section{Formulation of the problem and its solution}

We consider a spherical nanoheterosystem. Its model is presented in figure 1. It is formed by a nanocrystal of radius $a$ with dielectric permittivity $\varepsilon_{1}$, which is placed in a matrix with dielectric permittivity $\varepsilon_{2}$. At the separation boundary of the media there is a transitional layer with the thickness $L$, where dielectric permittivity is a function of $r$ coordinate. In the centre of this spherical QD there is a univalent (bivalent) impurity. The univalent (bivalent) impurity stands for an electro-neutral system with one (two) electrons.

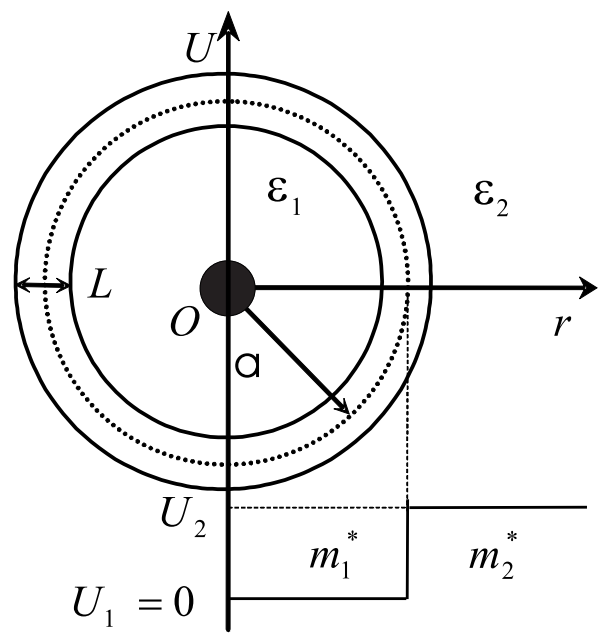

Figure 1. Schematic diagram showing a model of quantum system. 
A point charge Ze placed at $\mathbf{r}_{\mathbf{0}}$, induced the potential $\Phi\left(\mathbf{r}, \mathbf{r}_{\mathbf{0}}\right)$ at point $\mathbf{r}$. The potential can be found from the Poisson equation

$$
\nabla^{2} \Phi\left(\mathbf{r}, \mathbf{r}_{0}\right)+\frac{\mathrm{d}}{\mathrm{d} r}[\ln \varepsilon(r)] \frac{\partial}{\partial r}\left[\Phi\left(\mathbf{r}, \mathbf{r}_{0}\right)\right]=-\frac{4 \pi \mathrm{Z} e}{\varepsilon(r)} \delta\left(\mathbf{r}, \mathbf{r}_{0}\right)
$$

where $\delta\left(\mathbf{r}, \mathbf{r}_{\mathbf{0}}\right)$ is Dirac delta function. The solution of this equation allows us to find the interaction energy of $-e$ electron and the ion of a donor, interelectron interaction as well as the potential energy of interaction of each particle with polarization charges at the interface.

By solving the Poisson equation (1) one can derive an expression that describes the interaction of i-th electron with the impurity ion located in the quantum dot centre:

$$
W\left(r_{i}\right)=-e \cdot \Phi\left(r_{i}\right)=-Z e^{2}\left\{\begin{array}{l}
\left(\varepsilon_{1}-\varepsilon_{2}\right) /\left(\varepsilon_{1} \varepsilon_{2} a\right)+1 /\left(\varepsilon_{1} r_{i}\right), \quad r_{i}<a, \\
1 /\left(\varepsilon_{2} r_{i}\right), \quad r_{i} \geqslant a .
\end{array}\right.
$$

The term $-Z e^{2}\left(\varepsilon_{1}-\varepsilon_{2}\right) /\left(\varepsilon_{1} \varepsilon_{2} a\right)$ in (2) arises due to the existence of the interface, and all the rest terms are purely Coulomb that describe the interaction among particles in the first $\varepsilon_{1}$ or the second $\varepsilon_{2}$ medium. The expression of interelectronic interaction is given by:

$$
W\left(r_{1}, r_{2}\right)=e^{2} \cdot \begin{cases}\frac{\varepsilon_{1}-\varepsilon_{2}}{\varepsilon_{1}} \sum_{n=0}^{\infty} \frac{n+1}{\varepsilon_{1} n+\varepsilon_{2}(n+1)} \frac{r_{1}^{n} r_{2}^{n}}{a^{2 n+1}} P_{n}(\cos (\theta))+ & r_{1}<a, r_{2}<a, r_{2}<r_{1}, \\ +\frac{1}{\varepsilon_{1}} \sum_{n=0}^{\infty} \frac{r_{2}^{n}}{r_{1}^{n+1}} P_{n}(\cos (\theta)), & \\ \frac{\varepsilon_{1}-\varepsilon_{2}}{\varepsilon_{1}} \sum_{n=0}^{\infty} \frac{n+1}{\varepsilon_{1} n+\varepsilon_{2}(n+1)} \frac{r_{1}^{n} r_{2}^{n}}{a^{2 n+1}} P_{n}(\cos (\theta))+ & r_{1}<a, r_{2}<a, r_{1}<r_{2}, \\ +\frac{1}{\varepsilon_{1}} \sum_{n=0}^{\infty} \frac{r_{1}^{n}}{r_{2}^{n+1}} P_{n}(\cos (\theta)), & r_{1}<a, r_{2}>a, \\ \sum_{n=0}^{\infty} \frac{2 n+1}{\varepsilon_{1} n+\varepsilon_{2}(n+1)} \frac{r_{1}^{n}}{r_{2}^{n+1}} P_{n}(\cos (\theta)), & r_{1}>a, r_{2}<a, \\ \sum_{n=0}^{\infty} \frac{2 n+1}{\varepsilon_{1} n+\varepsilon_{2}(n+1)} \frac{r_{2}^{n}}{r_{1}^{n+1}} P_{n}(\cos (\theta)), & r_{1}>a, r_{2}>a, r_{2}<r_{1}, \\ \frac{\varepsilon_{2}-\varepsilon_{1}}{\varepsilon_{2}} \sum_{n=0}^{\infty} \frac{n}{\varepsilon_{1} n+\varepsilon_{2}(n+1)} \frac{a^{2 n+1}}{r_{1}^{n+1} r_{2}^{n+1}} P_{n}(\cos (\theta))+ & \\ +\frac{1}{\varepsilon_{2}} \sum_{n=0}^{\infty} \frac{r_{2}^{n}}{r_{1}^{n+1}} P_{n}(\cos (\theta)), & r_{1}>a, r_{2}>a, r_{1}<r_{2}, \\ \frac{\varepsilon_{2}-\varepsilon_{1}}{\varepsilon_{2}} \sum_{n=0}^{\infty} \frac{n}{\varepsilon_{1} n+\varepsilon_{2}(n+1)} \frac{a^{2 n+1}}{r_{1}^{n+1} r_{2}^{n+1}} P_{n}(\cos (\theta))+ & \\ +\frac{1}{\varepsilon_{2}} \sum_{n=0}^{\infty} \frac{r_{1}^{n}}{r_{2}^{n+1}} P_{n}(\cos (\theta)), & \end{cases}
$$

$P_{n}(x)$ are Legendre polynomials.

The electron will induce polarization charges at the separation boundary of the media. The corresponding energy of interaction of electron-polarization charges will be determined

$$
\begin{aligned}
V\left(r_{i}\right) & =\frac{\mathrm{e}^{2} \gamma}{4 \varepsilon\left(r_{i}\right)} \int_{0}^{\infty} \mathrm{d} r\left[\operatorname{th}\left(\frac{r-a}{L}\right)+\frac{r}{L} \operatorname{sech}^{2}\left(\frac{r-a}{L}\right)\right] \frac{1}{r^{2}-r_{i}^{2}} \\
\varepsilon\left(r_{i}\right) & =\frac{\varepsilon_{1}+\varepsilon_{2}}{2}\left[1-\gamma \cdot \operatorname{th}\left(\frac{r_{i}-a}{L}\right)\right] \\
\gamma & =\frac{\varepsilon_{1}-\varepsilon_{2}}{\varepsilon_{1}+\varepsilon_{2}}
\end{aligned}
$$

if a transitional layer of the thickness $L$ is taken into account, in which dielectric permittivity ranges between the value $\varepsilon_{1}$ of one medium to $\varepsilon_{2}$ of the other medium [17]. If it is neglected $(L=0)$, then the energy, which is also called the potential of forces of electrostatic images, has a non-physical divergence at $r=a[16]$ :

$$
V\left(r_{i}\right)=\frac{e^{2} \gamma}{2 a \varepsilon_{1}}\left[\frac{a^{2}}{a^{2}-r_{i}^{2}}+\frac{\varepsilon_{1}}{\varepsilon_{2}} F\left(1, \frac{\varepsilon_{2}}{\varepsilon_{1}+\varepsilon_{2}}, \frac{\varepsilon_{2}}{\varepsilon_{1}+\varepsilon_{2}}+1,\left(\frac{r_{i}}{a}\right)^{2}\right)\right], \quad \text { if } \quad r_{i}<a,
$$




$$
V\left(r_{i}\right)=\frac{e^{2} \gamma}{2 a \varepsilon_{2}}\left[\frac{a^{2}}{a^{2}-r_{i}^{2}}+\left(\frac{a}{r_{i}}\right)^{2} F\left(1, \frac{\varepsilon_{2}}{\varepsilon_{1}+\varepsilon_{2}}, \frac{\varepsilon_{2}}{\varepsilon_{1}+\varepsilon_{2}}+1,\left(\frac{a}{r_{i}}\right)^{2}\right)\right], \quad \text { if } \quad r_{i} \geqslant a,
$$

where $F(x, y, z, u)$ is confluent hypergeometric function.

The plots of the dependence of the potentials $(4),(7)-(8)$ on the distance to the centre of coordinates for nanoheterosystem $\beta$ - HgS/CdS, when QD radius is $50 \AA$, are presented in figure 2 .

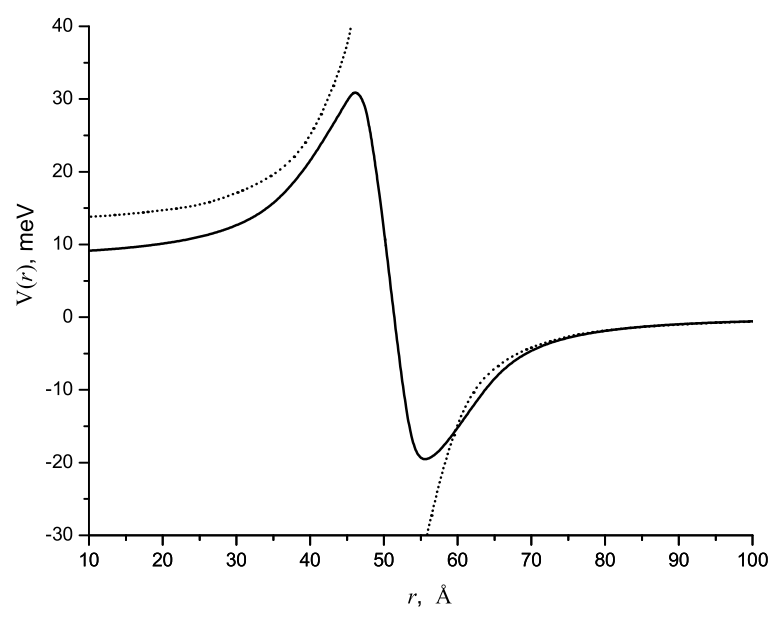

Figure 2. The potential energy of electron-induced charges at the separation boundary of a nanoheterosystem $\beta$-HgS/CdS with a transitional layer $L=5 \AA$ (continuous curve) and $L=0 \AA$ (dotted curve).

Let there be a univalent impurity in the center of a spherical quantum dot. The Hamiltonian of this system, using an effective mass approximation, will be written:

$$
\mathbf{H}=-\frac{\hbar^{2}}{2} \nabla \frac{1}{m^{*}(r)} \nabla+U(r)+W(r)+V(r)=\mathbf{H}_{0}(r)+W(r)+V(r),
$$

where

$$
Z=1, m^{*}(r)= \begin{cases}m_{1}^{*}, & r<a \\ m_{2}^{*}, & r \geqslant a\end{cases}
$$

is an effective mass of an electron in the corresponding crystal, $V(r)$ will have the form of (4) or (7)-(8). The potential energy due to the band mismatch (confinement potential) in a quantum dot is expressed by the formula:

$$
U(r)=\left\{\begin{array}{ll}
0, & r<a \\
U_{2}, & r \geqslant a
\end{array} \quad U_{2}>0\right.
$$

The Schrödinger equation with Hamiltonian (9) was solved using the variational method. In order to choose a trial function, the problem of one particle (an electron) in both infinite and finite spherical potential wells is used. The ground state of a charge in a spherical potential well with infinitely high wells is described by the function

$$
g_{1 s}(r)=\sqrt{1 / 2 \pi a} \begin{cases}\sin (\pi r / a) / r, & r<a, \\ 0, & r \geqslant a,\end{cases}
$$

and for the model of a finite potential at the interface it has the form

$$
g_{1 s}(r)=\frac{1}{\sqrt{4 \pi}}\left\{\begin{array}{ll}
A_{1} \cdot \sin (k r) / r, & r<a, \\
A_{2} \cdot \exp (-\chi r) / r, & r \geqslant a,
\end{array}= \begin{cases}g_{1}(r), & r<a, \\
g_{2}(r), & r \geqslant a,\end{cases}\right.
$$


where

$$
k=\sqrt{\frac{2 m_{1}^{*}}{\hbar^{2}} E_{0}}, \quad \chi=\sqrt{\frac{2 m_{2}^{*}}{\hbar^{2}}\left(U_{2}-E_{0}\right)} .
$$

$E_{0}$ is the energy of the particle ground state, constants $A_{1}$ and $A_{2}$ are defined from boundary conditions and from a normalizing condition

$$
\begin{aligned}
\left.g_{1}(r)\right|_{r=a} & =\left.g_{2}(r)\right|_{r=a}, \\
\left.\frac{1}{m_{1}^{*}} \frac{d}{d r} g_{1}(r)\right|_{r=a} & =\left.\frac{1}{m_{2}^{*}} \frac{d}{d r} g_{2}(r)\right|_{r=a}, \\
\int d \vec{r} \cdot\left|g_{1 s}(r)\right|^{2} & =1 .
\end{aligned}
$$

We consider QDs of small sizes, for which the kinetic energy of a particle is much greater than the potential energy. That is why a trial variational function for the infinite potential model at the separation boundary was chosen in the form

$$
\Psi(r)=N_{1} g_{1 s}(r) \exp (-\delta r) .
$$

The exponent in (16) describes the interaction of an electron and positively charged impurity ion, $\delta$ is a variational parameter, $N_{1}$ is a normalizing constant. For the finite potential model at the interface, a trial function is written

$$
\Psi(r)= \begin{cases}B_{1} \cdot g_{1}(r) \exp [-\alpha r], & r<a, \\ B_{2} \cdot g_{2}(r) \exp [-\beta r], & r \geqslant a .\end{cases}
$$

Values $\beta, B_{2}$ are defined from the boundary conditions for function $\Psi(r)$

$$
\begin{aligned}
\left.B_{1}\left(g_{1}(r) \exp (-\alpha r)\right)\right|_{r=a} & =\left.B_{2}\left(g_{2}(r) \exp (-\beta r)\right)\right|_{r=a}, \\
\left.\frac{B_{1}}{m_{1}^{*}} \frac{\mathrm{d}}{\mathrm{d} r}\left(g_{1}(r) \exp (-\alpha r)\right)\right|_{r=a} & =\left.\frac{B_{2}}{m_{2}^{*}} \frac{\mathrm{d}}{\mathrm{d} r}\left(g_{2}(r) \exp (-\beta r)\right)\right|_{r=a} .
\end{aligned}
$$

It is found that

$$
B_{2}=B_{1} \exp [a(\beta-\alpha)], \quad \beta=\frac{m_{2}^{*}}{m_{1}^{*}} \alpha .
$$

According to the Ritz variational principle, the energy of the system is determined by minimizing the functional $E(\alpha)$

$$
E(\alpha)=\frac{\langle\Psi|\mathbf{H}| \Psi\rangle}{\langle\Psi \mid \Psi\rangle} .
$$

Let there be a bivalent impurity in the center of a spherical QD. The Hamiltonian of this system in the effective mass approximation will be written

$$
\mathbf{H}\left(r_{1}, r_{2}\right)=\mathbf{H}_{0}\left(r_{1}\right)+\mathbf{H}_{0}\left(r_{2}\right)+W\left(r_{1}, r_{2}\right)+V\left(r_{1}\right)+V\left(r_{2}\right)+W\left(r_{1}\right)+W\left(r_{2}\right),
$$

where $\mathbf{H}_{\mathbf{0}}\left(\mathbf{r}_{\mathbf{i}}\right)$ is the Hamiltonian of a particle in a spherical potential well without impurity, $i=1,2, Z=2$. Here the interelectron interaction, interaction between each electron and ion of an impurity, interaction between each electron and "intrinsic" polarization charges are taken into account. The energy of the system is counted from the energy of interaction between an ion of impurity and its "intrinsic" induced charges.

The energies and wave functions are the solutions of the stationary Schrödinger equation

$$
\mathbf{H}\left(r_{1}, r_{2}\right) \Psi\left(r_{1}, r_{2}\right)=E \Psi\left(r_{1}, r_{2}\right) .
$$

It is not solved exactly. Therefore, the variational Ritz method was used to determine the ground state. It is seen from the Hamiltonian that it is enough to consider the wave function which depends 
on $r_{1}, r_{2}$. Thus, the solutions of the problem of a particle in a spherical quantum well with finite and infinite wells were used. The wave function for a model of an infinite potential at interface can be written

$$
\Psi\left(r_{1}, r_{2}\right)=N_{2} \cdot g_{1 s}\left(r_{1}\right) \cdot g_{1 s}\left(r_{2}\right) \cdot \exp \left[-\delta\left(r_{1}+r_{2}\right)\right]
$$

For the model of a finite potential at the separation boundary of media the wave function will be as follows:

$\Psi\left(r_{1}, r_{2}\right)=\left[\left\{\begin{array}{ll}B_{1} \cdot g_{1}\left(r_{1}\right) \exp \left[-\alpha r_{1}\right], & r_{1}<a \\ B_{2} \cdot g_{2}\left(r_{1}\right) \exp \left[-\beta r_{1}\right], & r_{1} \geqslant a .\end{array}\right] \cdot\left[\left\{\begin{array}{ll}B_{1} \cdot g_{1}\left(r_{2}\right) \exp \left[-\alpha r_{2}\right], & r_{2}<a \\ B_{2} \cdot g_{2}\left(r_{2}\right) \exp \left[-\beta r_{2}\right], & r_{2} \geqslant a .\end{array}\right]\right.\right.$.

In a similar manner, by minimizing a functional of form (20) for a presented system one can find the energy of electrons.

\section{The analysis of the obtained results}

The results were obtained for a univalent and a bivalent donor located in the centre of a QD. We assume the basic parameters of crystals that form the heterostructure to be equal to the volume crystal parameters (table 1).

Table 1. Parameters of the nanoheterostructure $\beta$-HgS/CdS.

\begin{tabular}{|c|c|c|c|c|c|c|}
\hline$m_{1} / m_{0}$ & $m_{2} / m_{0}$ & $\varepsilon_{1}$ & $\varepsilon_{2}$ & $U_{2}, \mathrm{meV}$ & $E_{1}^{g}, \mathrm{meV}$ & $E_{2}^{g}, \mathrm{meV}$ \\
\hline 0.036 & 0.2 & 11.36 & 5.82 & 1200 & 500 & 2500 \\
\hline
\end{tabular}

Figure 3 shows the dependencies of the ground state energy for univalent and bivalent donors in the centre of the nanoheterostructure $\beta-\mathrm{HgS} / \mathrm{CdS}$ taking into account the finite and infinite band mismatch and the interaction of the particles and the interface of the heterostructure.

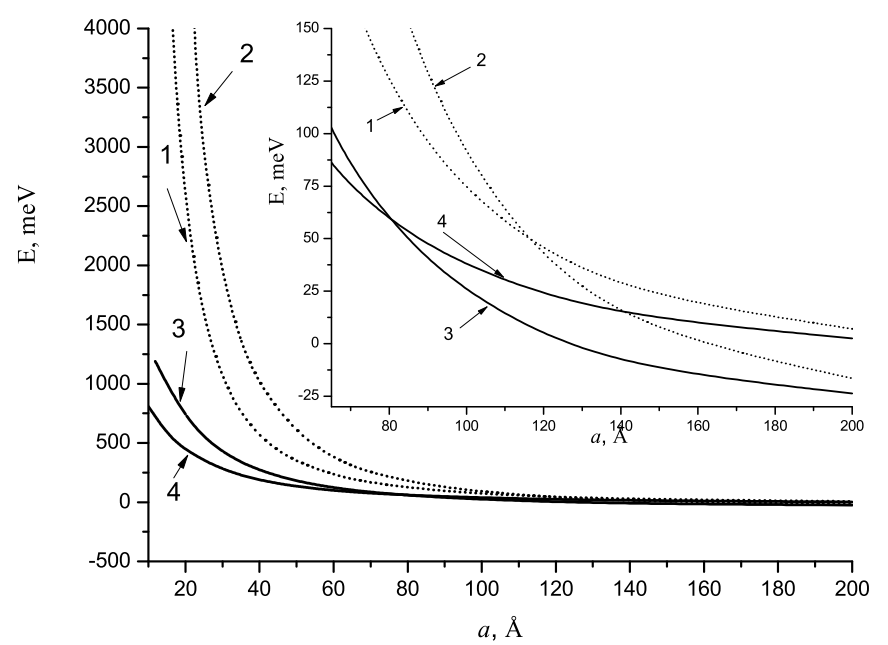

Figure 3. The energy of univalent $(1,4)$ and bivalent $(2,3)$ donors in a spherical QD with infinite $(1,2)$ and finite $(3,4)$ potential at the separation boundary of the media.

In order to calculate the interaction of particles and the interface of the heterostructure for a model of an infinite band mismatch equations (7)-(8) were used, and for a finite one equation (4) was used. The magnitude of an intermediate layer was assumed $L=5 \AA$. 
It is seen from the plots that the energy of a QD almost does not depend on QD radius at large radii. The smooth energy change is caused by the weak effect of the potential well and the surface on the energy levels, and the energy is mainly defined by the interaction of electrons and an ion of the impurity. A certain difference is observed between the energies obtained by using models of a finite and an infinite well.

When the QD radius decreases, the confined potential (11) and polarization substantially effects the energy of electrons. The leads to the increase of energy of the system. For the model of an infinite confinement, the energies of a univalent and a bivalent donors grow faster than those for a finite potential. Also, as a result of interelectronic interaction, the increase of the energy of a bivalent impurity prevails over the rise of the energy of a univalent impurity within these models.

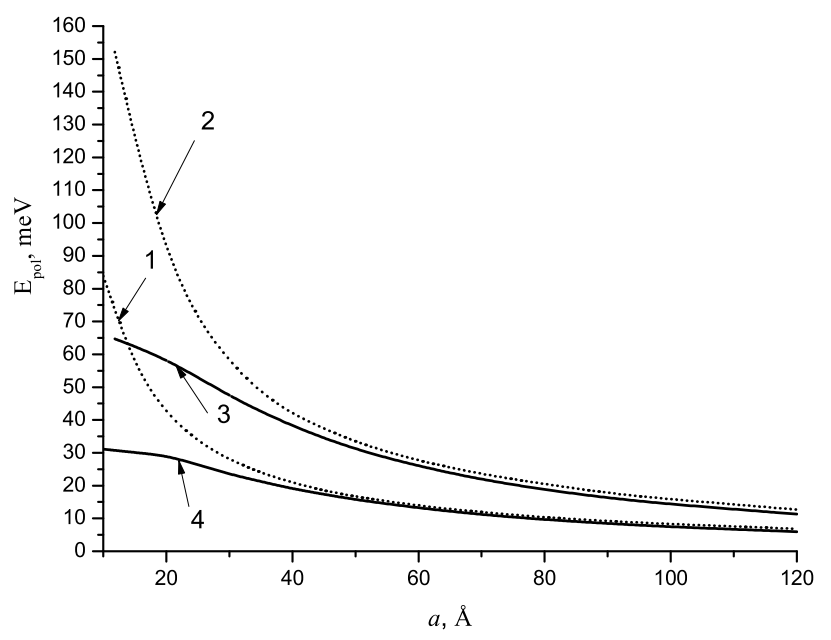

Figure 4. The energy caused by polarization charges at the separation boundary of the media of a univalent $(1,4)$ and a bivalent $(2,3)$ donors in a spherical QD with finite $(3,4)$ and infinite $(1,2)$ potentials at the interface.

To define a contribution given by polarization charges at the interface on the energy of a nanoheterostructure, the calculation of the energy of univalent and bivalent donors was carried out in a spherical QD without considering the interaction between electrons and polarization charges. The difference between the total energy and the energy without induced charges at the interface of heterostructure will show the effect of induced charges at the separation boundary of the media on the energy of the system (figure 4 ). This quantity is denoted by $E_{\mathrm{pol}}$. It is worth mentioning that in the considered nanoheterostructure, the dielectric permittivity of the matrix is considerably less than that of a QD. Therefore, for charges in a QD the $V\left(r_{i}\right)$ is a positive function, which increases while approaching to the surface. Thus, the contribution to the energies of particles caused by the presence of the surface polarization is positive.

The energy of the system increases when a radius of a QD decreases, but if a QD radius is large, the effect of polarization charges is small, because the rise of a QD radius reduces the effect of the surface on electrons. From figure 4 it is also seen that $E_{\text {pol }}$ for a bivalent donor is greater than that for a univalent donor. This is explained by the fact that a bivalent donor has two electrons, each of them enhancing its energy due to the interaction with the induced charges, and a univalent donor has only one electron. Therefore, the energy caused by polarization charges of a bivalent donor is two times greater than that of a univalent. Since the potential energies of interaction between electron and induced charges at the interface are different at small QD radii, it is seen from figure 4 that $E_{\mathrm{pol}}$ for the models of finite and infinite potentials at small QD radii differ substantially. The increase of the energy $E_{\text {pol }}$ with a decrease of a QD radius is caused by the growing effect of the surface on the electrons. 


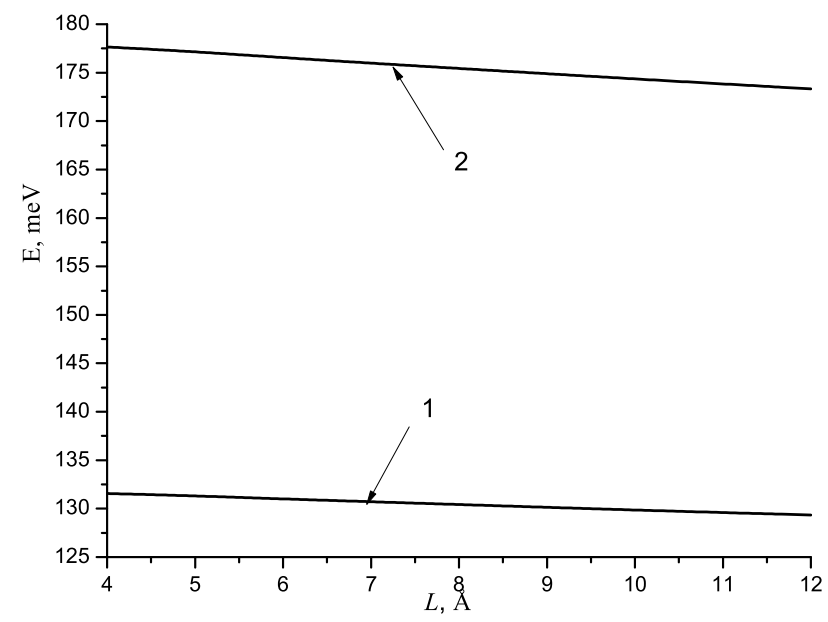

Figure 5. The dependence of a univalent (1) and a bivalent(2) donor energy on the value of a nanoheterostructure intermediate layer with radius $a=50 \AA$.

In the present work, the energy of univalent and bivalent donors is calculated as a function of the thickness of an intermediate layer. This dependence is shown in figure 5 . It is seen from figure 5 that the reduction of an intermediate layer results in an increase of the energy. This is explained by the fact that with reduction of $L$, the potential (4) approaches the potential (7)-(8) that is a confinement of the growth of electrons, and thus the energy is enhanced.

Thus, in the present work, the electron energy dependencies of a univalent and a bivalent impurity are determinated as functions of a QD radius. The effect of a transitional layer $L$, where dielectric permittivity is a function of coordinates on the energy of donors is studied. The results showed that the reduction of a transitional layer enhances the energy of the system. The contribution of polarization charges on the energy of a nanoheterosystem is defined. It is found that the contribution of a bivalent donor is two times greater than that of a univalent donor. The calculation showed that in the range of small QD radii the effect of the induced charges on the energy of a nanostructure is essential. 


\title{
References
}

1. Jia-Zin Zhu, Phys. Rev. B, 1989, 39, 8780.

2. Jia-Zin Zhu, Phys. Rev. B, 1990, 41, 6001.

3. Chu D.S., Hsiao C.M., Mei W.N., Phys. Rev. B, 1992, 46, 3898.

4. Riberio F.J., Latge A., Phys. Rev. B, 1994, 50, 7.

5. Jia-Zin Zhu, Phys. Rev. B, 1994, 50, 4497.

6. Deng Z.Y., Guo J., Lai T., Phys. Rev. B, 1994, 50, 5736.

7. Krevchik V.D., Zaitsev R.V., Evstifeev V.V., Fiz. Tekhn. Polupr., 2000, 34, 1244 (in Russian).

8. Krevchik V.D., Lewashov A.V., Fiz. Tekhn. Polupr., 2002, 36, 216 (in Russian).

9. Chun-Ching Yang, Li-Chi Liu, Shih-Hsin Chang, Phys. Rev. B, 1998, 58, 1954.

10. Tkach M.V., Holovatsky V.A., Berezovsky Y.M., Phys. Chem. of Sol. State, 2003, 4, 213 (in Ukrainian).

11. Aleshkin V.Ya., Gavrilenko L.V., Fiz. Tverd. Tela, 2005, 39, 63 (in Russian).

12. Boichuk V.I., Nitsovich V.M., Tkach N.V., Fiz. Tverd. Tela, 1980, 22, 669 (in Russian).

13. Boichuk V.I., Bilynskii I.V., Phys. Stat. Sol., 1992, 174, 463.

14. Boichuk V.I., Voitsekhivska O.N., Holovatsky V.A., Tkach M.V., Fiz. Tverd. Tela, 1995, 37, 861 (in Russian).

15. Nakamura T., J. Phys. Soc. Japan 1983, 52, 973.

16. Efremov N.A., Pokutnyi S.I., Fiz. Tverd. Tela, 1985, 27, 48 (in Russian).

17. Boichuk V.I., Kubai R.Yu., Fiz. Tverd. Tela, 2001, 43, 226 (in Russian).

18. Holovatsky V.A., Ukr. J. Phys., 2006, 51, 207.

\section{Вплив поляризаційних зарядів на енергію одновалентного та двовалентного донора у сферичній квантовій точці}

\author{
В.І.Бойчук ${ }^{1}$, І.В.Білинський ${ }^{1}$, Р.Я.Лешко ${ }^{1}$ \\ ${ }^{1}$ Кафедра теоретичної фізики, Дрогобицький державний педагогічний університет ім. І. Франка, \\ Дрогобич 82100, вул. Стрийська, 3 \\ Отримано 4 березня 2008 р., в остаточному вигляді - 17 липня 2008 р.
}

Для сферичної наногетероструктури $\beta$-HgS/CdS у наближенні ефективної маси варіаційним способом обчислено енергію електронів одновалентної та двовалентної домішки як функцію радіуса квантової точки у випадку скінченної та нескінченної потенціальної ями. Досліджено вплив поляризаційних зарядів, що виникають на границі поділу середовищ, з врахуванням існування проміжного шару, де діелектрична проникність залежить від координат, і його відсутністю.

Ключові слова: енергія, домішка, поляризаційні заряди, потенціальна яма

PACS: 71.55.-i, 73.21.La, 79.60.JV 
